\title{
High-Precision MRI Reconstruction Algorithm for 3D Sphere Packings
}

\author{
R. Balzan ${ }^{1,3}$ - A. L. Sellerio ${ }^{2,4,5}$ - D. Mari ${ }^{2}$. \\ A. Comment ${ }^{1}$
}

Received: 16 January 2015/Published online: 18 April 2015

(c) Springer-Verlag Wien 2015

\begin{abstract}
Packings of granular materials are complex systems consisting of large sets of particles interacting via contact forces. Their internal structure is interesting for several theoretical and practical reasons, especially when the model system consists in a large amount (up to $10^{5}$ ) of identical spheres. We herein present a method to process three-dimensional water density maps recorded in wet granular packings of mm-size spheres by magnetic resonance imaging (MRI). Packings of spheres with highly monodispersed diameter are considered and the implementation of an ad hoc reconstruction algorithm tailored for this feature allows for the determination of the position of each single sphere with an unprecedented precision (with respect to the scale of the system) while ensuring that all spheres are identified and no non-existing sphere is introduced in the reconstructed packing. The reconstruction of a $0.5 \mathrm{~L}$ sample containing about $2 \times 10^{4}$ spheres is presented to demonstrate the robustness of the method.
\end{abstract}

\section{Introduction}

Granular packings are excellent model systems for the study of many open problems in rheology and non-equilibrium statistical mechanics [1]. They are loosely defined as large sets of macroscopic particles that interact only via contact forces such as

R. Balzan

balzan.riccardo@parisdescartes.fr

1 Institute of Physics of Biological Systems, EPFL, 1015 Lausanne, Switzerland

2 Institute of Condensed Matter Physics, EPFL, 1015 Lausanne, Switzerland

3 Laboratoire de Chimie et Biochimie Toxicologiques et Pharmacologiques UMR8601, Universitè Paris Descartes, CNRS, PRES Paris Sorbonne Citè, 75006 Paris, France

4 Present Address: IENI-CNR, 20125 Milan, Italy

5 Dipartimento di Fisica, Università degli Studi di Milano, 20133 Milan, Italy 
Hertzian forces and dry friction [2, 3], and via inelastic collisions [4]. However, the apparent simplicity of the forces that govern their static and dynamic properties hides an underlying complexity that one still fails to understand [5].

Studies on the geometrical properties of arrangements of spheres date back to Kepler [6]. In modern times, the problem has been tackled also from an experimental point of view [7-10] and has recently seen a renewed interest [5, 11]. In addition to theoretical and rheological approaches, the determination of the static structure of granular packings is of crucial importance in the comprehension of their properties.

To date, the largest three-dimensional reconstruction from tomographic projections of granular packings has been obtained using X-ray computed tomography and a Fourier space reconstruction algorithm [12]. Although this method allows for the determination of the structure of packings composed of up to $1.5 \times 10^{5} 1-\mathrm{mm}$ spheres, neither the identification of each single sphere nor the absence of nonexisting spheres incorporated into the reconstruction can be guaranteed. The importance of a flawless reconstruction method can be highlighted by the following observation: failing to identify 1 out of 1000 spheres or mistakenly introducing a non-existing sphere in a sample of 1000 spheres leads to a computational error on the packing density that corresponds to about $1 \%$ of the full density range inherent to random sphere packings, comprised between the limits of random close packing (RCP) [13] and random loose packing (RLP) [14].

More direct approaches [15] consist in acquiring extremely high-resolution data, and then apply simple image analysis filters (threshold segmentation and erosion), but this requires long acquisition times and computationally intensive image postprocessing.

We herein present a robust and precise method based on magnetic resonance imaging (MRI) to determine the exact position of sphere centers in wet granular packings composed of $2 \times 10^{4}$ spheres. This reconstruction method was previously used to study fine structural details of sphere packings [16]. The aim of this study is to describe in detail the algorithm and the method on which the reconstruction procedure is based, and to highlight its wide applicability for studying granular materials.

\section{Materials and Methods}

A standard MRI method, namely gradient echo (GRE) imaging [17], was used to acquire tomographic projections. The projections were processed on a standard desktop computer to determine the three-dimensional coordinates of all sphere centers and reconstruct the packings.

The studied packings were composed by 3-mm nominal diameter plastic spheres (polyoxymethylene, R.P.G. International, Italy).

To obtain a precise determination of the sample density, we measured the exact average volume and standard deviation $V_{\mathrm{s}}=14.348 \pm 0.07 \mathrm{~mm}^{3}$ of the plastic spheres using Archimede's method. This corresponds to an average sphere diameter of $d=3.0148 \mathrm{~mm}$ with a standard deviation of $\sigma_{\mathrm{d}}=1.65 \times 10^{-3} \mathrm{~d}$. The mismatch 
between the nominal $14.137 \mathrm{~mm}^{3}$ and the measured $14.348 \mathrm{~mm}^{3}$ volume is about $1.5 \%$ and it was taken into account in all calculations, including the determination of the packing density.

The packing preparation consisted in soaking the plastic spheres in a $\mathrm{CuSO}_{4}^{-}$ doped aqueous solution; this operation was necessary to avoid formation of air bubbles in the following procedures. The spheres were subsequently slowly poured in a vertical cylinder previously filled with the same solution (Fig. 1a). The density of the solution was not matched to the sphere density. The $\mathrm{CuSO}_{4}$ concentration was chosen to set the nuclear magnetic resonance (NMR) longitudinal relaxation time $T_{1}$ and transverse relaxation time $T_{2}$ of protons to $T_{1} \sim T_{2} \sim 15 \mathrm{~ms}$.

The sample was vertically vibrated using colored noise agitation, with a flat spectrum in the frequency range $40-1000 \mathrm{~Hz}$ and vanishing amplitude at other frequencies. We agitated with average acceleration modulus $\sqrt{\left\langle\ddot{z}^{2}\right\rangle} \sim 2.5 \mathrm{~g}$, and kept it still (no convection) for 30 min (aging procedure).

The sample was scanned in a head-only $7 \mathrm{~T}$ human MRI scanner (Siemens, Erlangen, Germany) using a birdcage volume coil designed to accommodate a human head. The three-dimensional images were acquired using a GRE imaging sequence (repetition time $T_{\mathrm{R}}=5 \mathrm{~ms}$, echo time $T_{\mathrm{E}}=3.79 \mathrm{~ms}$, resolution $0.2 \mathrm{~mm}$ isotropic, total acquisition time $\sim 6 \mathrm{~h}$ ). The raw data consisted in isotropic threedimensional gray scale images mapping the water proton density in each voxel (Fig. 2a).

Image processing was performed using a custom-made $\mathrm{C}$ program running on a standard dual-core desktop PC. The program takes a three-dimensional image as input, performs the three processing steps described in detail in the following section, and outputs a list containing the three-dimensional position associated with each identified sphere. Average running time for the reconstruction of a $0.5 \mathrm{~L}$ sample with about $2 \times 10^{4}$ spheres was $1 \mathrm{~h}$, scaling roughly linearly with the volume. An example of reconstructed packing is shown in Fig. 1b.
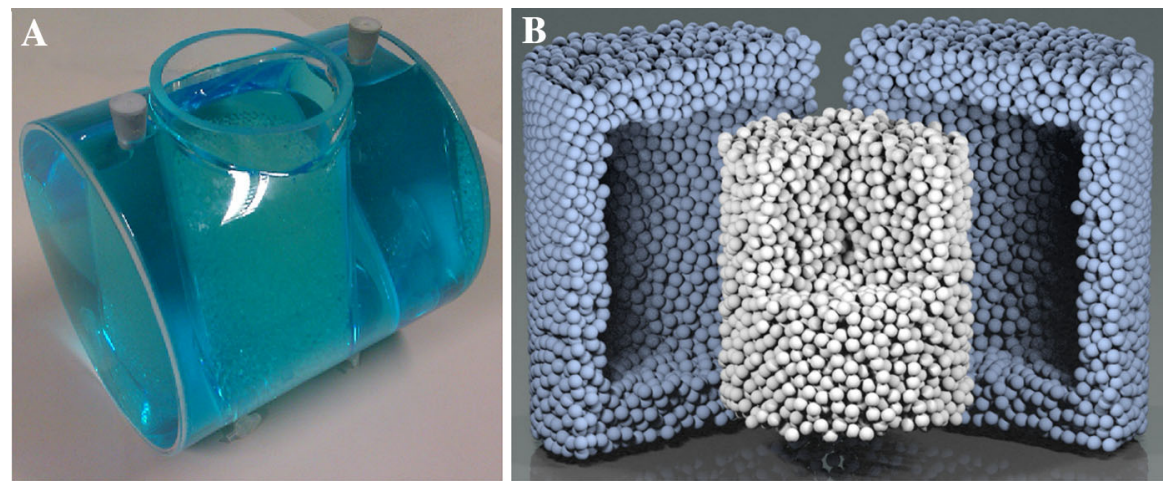

Fig. 1 a Granular packing inside the phantom used for the tomographic measurements; $\mathbf{b}$ example of 3D reconstructed packing. The outer spheres close to the container walls (light blue spheres) are discarded from the analysis to avoid boundary effects 

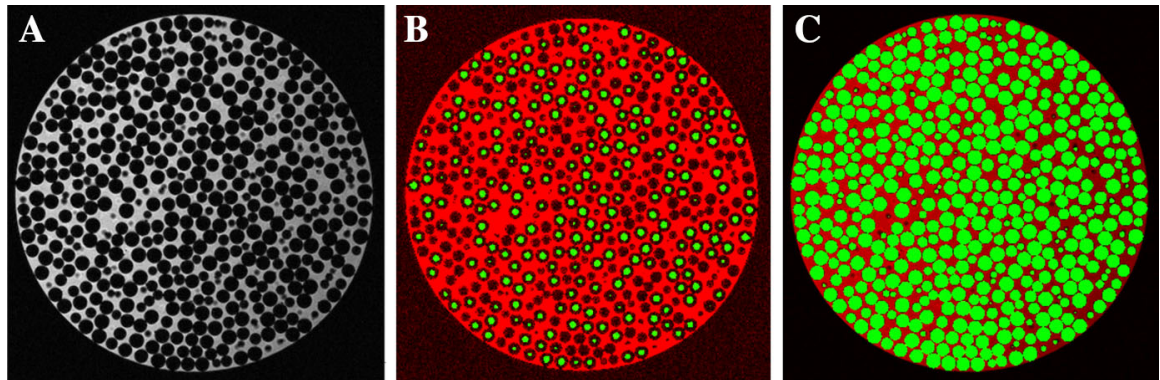

Fig. 2 Example of an acquired and processed horizontal slice. a Acquired raw image (gray scale); b 2D projection of the transformed image after step II of the reconstruction algorithm (green) superimposed onto the raw image (red); $\mathbf{c} 2 \mathrm{D}$ projection of the reconstructed sample (green) superimposed onto the raw image $(r e d)$

\subsection{Reconstruction Algorithm}

For each sample, we obtained a high contrast-to-noise ratio (CNR) 3D image consisting in a matrix $\boldsymbol{I}(x, y, z)$ containing the water proton density measured in each voxel (Fig. 2a). The reconstruction algorithm consists of three steps:

- I Construction of a 3D binary image Associate each image slice with a binary proton density map by forcing each voxel to be assigned either the value 1 if most of its volume is located inside a sphere or 0 if it mostly contains water.

- II Thresholded Hough transform filter This step sets to zero the proton density assigned to voxels containing contact points between adjacent spheres.

- III Individual sphere identification The last step uses the prior knowledge of the average sphere size and is based on the fact that, following steps I and II, the clusters of voxels representing individual spheres do not overlap.

\subsubsection{Construction of Binary Images}

The first step of the algorithm consists in identifying the voxels contained in the volume occupied by the spheres.

Although the CNR of the acquired images was high, their brightness was not uniform across the sample volume. To overcome this issue, we used a local threshold criterion to identify the voxels located inside the plastic spheres $[18,19]$. We defined a local threshold value $T(x, y, z)$ for each voxel as follows:

$$
T(x, y, z)=\left(\max \left[\boldsymbol{I}\left(x^{\prime}, y^{\prime}, z^{\prime}\right)\right]-\min \left[\boldsymbol{I}\left(x^{\prime}, y^{\prime}, z^{\prime}\right)\right]\right) t+\min \left[\boldsymbol{I}\left(x^{\prime}, y^{\prime}, z^{\prime}\right)\right],
$$

where $\max \left[\boldsymbol{I}\left(x^{\prime}, y^{\prime}, z^{\prime}\right)\right]$ and $\min \left[\boldsymbol{I}\left(x^{\prime}, y^{\prime}, z^{\prime}\right)\right]$ represent the best estimation, given the local brightness level, of the intensity of an entirely water-filled and an entirely sphere-filled voxel, respectively, and $t$ is a threshold parameter such as $0<t<1$. The local maxima and minima are defined with the condition $\left|\left(x-x^{\prime}, y-y^{\prime}, z-z^{\prime}\right)\right|<R_{\mathrm{T}}$, with $R_{\mathrm{T}}$ as the local threshold radius. There are thus 
two free parameters in Eq. 1, namely $t$ and $R_{\mathrm{T}}$. To compute a binary thresholded image, a Heaviside step function $\theta(\cdot)$ was used to obtain a matrix:

$$
\boldsymbol{I}_{\mathrm{t}}(x, y, z)=\theta(T(x, y, z)-\boldsymbol{I}(x, y, z)),
$$

in which all voxels identified as being within a sphere are assigned the value 1 .

$R_{\mathrm{T}}$ and $t$ are not strongly correlated and, due to the high CNR of the acquired data, these two parameters can be assigned values lying within a wide range without dramatically affecting the quality of the thresholding procedure. $R_{\mathrm{T}}$ has to be large enough to ensure that the local max and min functions span over both types of voxels, i.e., water-filled and sphere-filled voxels. This condition imposes a lower limit, namely $R_{\mathrm{T}}>d / 2$. On the other hand, if $R_{\mathrm{T}}$ is too large, spatial fluctuations in image brightness might affect the accuracy of the determination of the local maximum and minimum values. Considering these two constraints, the value $R_{\mathrm{T}}=$ $1.5 \mathrm{~d}$ was chosen. The natural choice for $t$ is 0.5 since it will lead to a preservation of the average local brightness of the image. Increasing $t$ will produce an increase in the apparent spheres size and blur the contact points between them. Conversely, decreasing $t$ will help separate the spheres but lead to loss of positive matches in voxels close to the sphere boundaries. However, for images with a high enough $\mathrm{CNR}$, as is the case for the herein presented acquisitions, it was found that the results are only weakly depending on the exact $t$ value as long as it stays within the range $0.25<t<0.75$. The value $t=0.5$ was chosen for all computations. Finally, the value of $\boldsymbol{I}_{t}(x, y, z)$ was set to zero for all voxels located outside the sample physical boundaries, which are known exactly from the geometry of the cylindrical container.

\subsubsection{Thresholded Hough Transform Filter}

Part of the clusters of voxels representing spheres in $\boldsymbol{I}_{t}$ may overlap due to the imperfect thresholding procedure described in step I. The aim of step II is thus to set to zero the voxels connecting neighboring clusters to obtain a matrix containing well-separated clusters. To do so, a real-space spherical Hough transform [20], and an additional threshold procedure was applied to $\boldsymbol{I}_{t}$. The matrix resulting from the spherical Hough transform has the same dimensions as $\boldsymbol{I}_{t}$ and is given by:

$$
H(x, y, z)=\frac{\sum \boldsymbol{I}_{t}\left(x^{\prime}, y^{\prime}, z^{\prime}\right) \theta\left(R_{\mathrm{H}}-\left|\left(x-x^{\prime}, y-y^{\prime}, z-z^{\prime}\right)\right|\right)}{\sum \theta\left(R_{\mathrm{H}}-\left|\left(x-x^{\prime}, y-y^{\prime}, z-z^{\prime}\right)\right|\right)},
$$

where $R_{\mathrm{H}}$ and $\theta\left(R_{\mathrm{H}}-\left|\left(x-x^{\prime}, y-y^{\prime}, z-z^{\prime}\right)\right|\right)$ are the radius and the kernel of the spherical Hough transform, respectively. The additional threshold procedure based on a Heaviside step function $\theta(\cdot)$ leads to a binary image described by the matrix:

$$
\boldsymbol{I}_{H}(x, y, z)=\theta(H(x, y, z)-h),
$$

where $h$ is an additional threshold parameter.

Unlike in step I, the two free parameters $R_{\mathrm{H}}$ and $h$ are strongly correlated. $R_{\mathrm{H}}$ has to be as small as possible to assign the maximum number of voxels to each cluster for insuring a high precision in the packing reconstruction, but large enough to obtain well-separated and nearly spherical clusters in $\boldsymbol{I}_{\mathrm{H}}$. The best compromise was 
empirically found to be $R_{H}=d / 3$. The natural choice for the threshold parameter would be $h \sim 1$ so that the only voxels that are set to 1 in $\boldsymbol{I}_{\mathrm{H}}$ are the ones for which almost the entire kernel of the Hough transform is filled with voxels set to 1 in $\boldsymbol{I}_{\mathrm{t}}$. We empirically determined that, for $R_{\mathrm{H}}=d / 3$, the packing reconstruction depends weakly on the exact $h$ value as long as it lies in the range $0.99>h>0.9$. The median of this range was chosen, i.e., $h=0.95$. With this choice of parameters, a binary image containing well-separated (distance of at least $\mathrm{d} / 4$ ) and nearly spherical voxel clusters were obtained (Fig. 2b).

\subsubsection{Individual Sphere Identification Algorithm}

The last part of the reconstruction procedure consists in calculating the center of mass of each cluster of voxels. To obtain a complete reconstruction, i.e., to ensure that all spheres are identified and no non-existing sphere is introduced into the packing by the algorithm, we implemented two checking procedures based on the properties of the clusters: first, to avoid the assignment of two neighboring spheres to a single sphere center, a cluster diameter must be at most $0.9 \mathrm{~d}$; second, to prevent isolated small clusters produced by noise to be identified as sphere, each cluster must at least contain a specified minimum number of voxels (this number was set to 50).

The algorithm sequentially analyzes all voxels, identifies the clusters and, once a cluster passes the checking procedures, its center of mass is computed and associated to the coordinates of a sphere center. Only the voxels assigned to a sphere are deleted from $\boldsymbol{I}_{\mathrm{H}}$ and the procedure repeated until all voxels have been considered. All remaining unassociated voxels contained in $\boldsymbol{I}_{\mathrm{H}}$ are plotted and superimposed to the original raw image to visually detect, a posteriori, the eventual presence of artifacts. The final output of this workflow consists in a dataset listing the spatial coordinates of each sphere center. To visually check the completeness of the reconstruction, the volume occupied by the reconstructed spheres in the datasets can be overlapped to the original raw image (Fig. 2c).

\section{Results}

To evaluate the robustness and the precision of the reconstructions, we performed two quality tests. The first test focused on the repeatability of the method and consisted in performing two sequential MRI acquisitions of the same sample to quantify the mismatch between two consecutive reconstructions. The second aimed at testing the accuracy of the method by computing and analyzing the two-body radial correlation function of a sample.

\subsection{Repeatability}

Two complete MRI datasets from the same sample were sequentially acquired and reconstructed using identical conditions and parameters. The same exact number of sphere centers was identified in the two datasets. Each identified sphere center from 

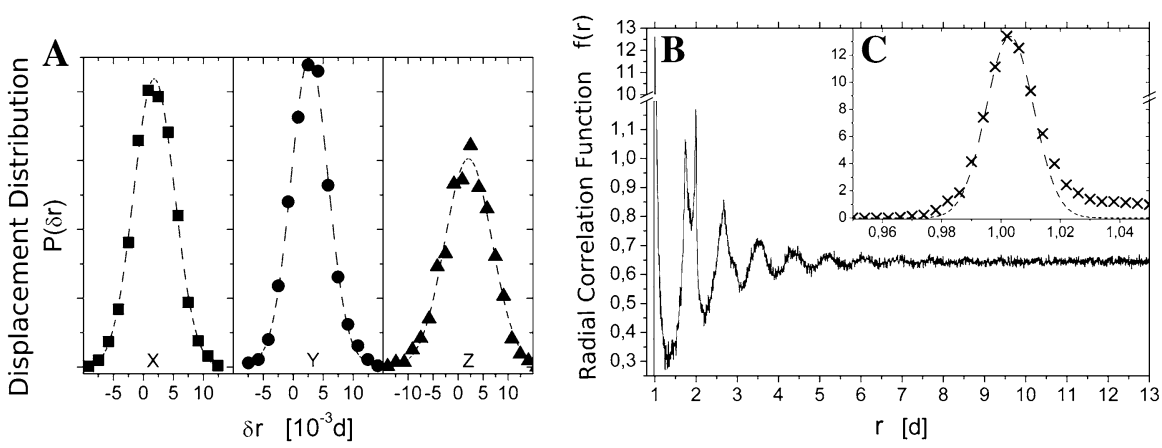

Fig. 3 a Displacement distribution along the three Cartesian directions in sequential reconstruction of the same sample. The standard deviations in units of sphere diameter are $\sigma_{X}=3.41 \times 10^{-3}$, $\sigma_{\mathrm{Y}}=3.09 \times 10^{-3}$ and $\sigma_{\mathrm{Z}}=4.7 \times 10^{-3}$. The dashed lines represent a Gaussian fit; b Two-body correlation function; c Detail of the peak around $r \sim 1$ with a Gaussian fit (dashed line) performed on the upper half of the peak. The computed standard deviation in units of sphere diameter is $\sigma_{\exp }=7 \times 10^{-3}$. In both graphs, the radial distance $(r)$ is expressed in units of diameter $(d)$

the first dataset was then associated to the corresponding sphere center in the second dataset. The differences in sphere center coordinates along the three Cartesian directions were determined (Fig. 3a).

The distribution of the displacements along the $x, y$ (horizontal) and $z$ (vertical) axes was considered separately and the resulting histograms were fitted with simple Gaussian functions to determine the standard deviation.

The displacement standard deviation was found to be in the range $3-5 \times 10^{-3} \mathrm{~d}$, leading to a repeatability error over the two datasets of $\varepsilon_{2} \sim 6 \times 10^{-3} \mathrm{~d}$, which is comparable to the sphere diameter standard deviation $\left(\sigma_{\mathrm{d}}=1.65 \times 10^{-3}\right)$. It must be highlighted that, although $\varepsilon_{2}$ is small and demonstrates the robustness of the method, the origin of the repeatability error may be partly due to a rearrangement (aging) of the packing, consequence of the motion induced by the gradient coil vibrations during MRI acquisitions.

\subsection{Correlation Function}

The radial correlation function $f(r)$ is a sensitive way to highlight geometrical properties of crystalline and amorphous structures. It is routinely used in granular material packings analysis.

Despite being an averaged quantity, $f(r)$ is extremely sensitive to the local properties of the sample.

It can be consistently used to assess the sensitivity of the reconstruction, provided the granular sample has a degree of polydispersion lower than the reconstruction error.

In our previous publication, and references therein, we discussed the properties of $f(r)$ and a method to reliably calculate the correlation function even in low-sized samples or sample fractions [16]. 
The spheres with centers located at a distance smaller than $3 \mathrm{~d}$ away from the container boundaries were discarded from the analysis to limit spurious boundary effects caused by the container walls (e.g., crystallization at boundaries). The twobody radial correlation function $f(r)$ is defined as the average number of centers located inside the spherical shell of thickness $\delta$ at distance $[r-\delta / 2, r+\delta / 2]$ from the center under consideration $N_{\delta}(r)$, normalized by the average sphere volume $V_{\mathrm{b}}$ and the spherical shell volume $V_{\delta}(r)$ :

$$
f(r)=\left\langle\frac{N_{\delta}(r) \cdot V_{b}}{V_{\delta}(r)}\right\rangle,
$$

A shell thickness of $\delta=10^{-3} \mathrm{~d}$ was chosen as an optimal trade-off value between high resolution and smoothness (Fig. 3b). The peak at $r \sim d$ represents the distribution of distances between spheres in direct contact (nearest neighbors). Its shape is essentially Gaussian, slightly skewed on the right side due to the presence of spheres almost, but not quite, in contact with the sphere under consideration. From the line width, it is possible to deduce the precision of the reconstruction. If we assume a perfect reconstruction and no correlation between nearest neighbors diameter, the line shape is expected to be Gaussian (except from the right side skewing) with a standard deviation $\sigma_{\mathrm{D}}$ given only by the dispersion in sphere diameter:

$$
\sigma_{\mathrm{D}}=\sigma_{\mathrm{d}} / \sqrt{2}=1.17 \times 10^{-3} \mathrm{~d},
$$

The line width of the peak increases with increasing reconstruction error $\sigma_{\mathrm{R}}$. If we assume that $\varepsilon_{R}$ is randomly distributed and not correlated with the nearest neighbor diameter, the line shape will still be Gaussian and the standard deviation is given by:

$$
\sigma_{\exp }=\sqrt{2 \varepsilon_{\mathrm{R}}^{2}+\frac{\sigma_{\mathrm{d}}^{2}}{2}},
$$

From a simple Gaussian fit of the $f(d)$ peak (Fig. 3c), it is possible to determine $\sigma_{\exp }$ and, inverting Eq. 7, the reconstruction error can be computed as follows:

$$
\varepsilon_{\mathrm{R}}=\sqrt{\frac{\sigma_{\exp }^{2}+\sigma_{\mathrm{d}}^{2} / 2}{2}},
$$

We observed that $5 \times 10^{-3} d<\sigma_{\exp }<8 \times 10^{-3} \mathrm{~d}$ for all recorded datasets [16]. From Eq. 8, we obtained a reconstruction error of $3 \cdot 10^{-3} d<\varepsilon_{\mathrm{R}}<5 \times 10^{-3} \mathrm{~d}$ which is on the same order as $\sigma_{\mathrm{d}}$ and $\varepsilon_{2}$.

\section{Discussion}

The MRI acquisition and reconstruction method herein presented exhibits two main benefits as compared to previous proposed schemes: first, the image acquisition time is short, allowing either recording rapid consecutive acquisitions to reduce statistical noise on a sample of given size or increasing the sample size, i.e., the number of 
spheres. Second, the high CNR together with the relatively high spatial resolution allows accurate determination of the sphere centers. This novel method gives unprecedented high-precision determination of the internal structure of granular packings, giving access to the observation of fine-grained details. The evaluation of the precision of the sample reconstruction allows for a direct quantification of the errors affecting the geometrical quantities extracted from the packing structure.

The small errors inherent to the reconstruction method allow computing, with high precision, the asymptotic value of $f(r)$ as $r$ becomes large, which provides a value for the granular density $\rho$ of the packing. This definition of granular density corresponds to the averaged long-range density of the system and can be compared to local densities defined, for instance, through the decomposition of the space in Voronoi polyhedra [21]. The herein presented reconstruction algorithm allows to observe the decay of the oscillatory behavior of $f(r)$ for $3<r<8$ and thus estimates with high accuracy the asymptotic value of $\rho$ even for relatively small samples or for partial volumes of a large sample [16].

A flawless reconstruction algorithm and a high monodispersion in sphere diameter are key features to obtain packings reconstructions with minimal errors in the determination of the sphere position.

For comparison, the precision of the reconstructed positions obtained in the state-of-the-art measurements presented in Aste et al. [12] was estimated from the number of voxels associated with each sphere, leading to questionably low values $\left(\sim 10^{-4} \mathrm{~d}\right)$, especially considering the relatively high scatter in sphere diameter $\left(4-5 \times 10^{-2} \mathrm{~d}\right)$. The uncertainty in the number of spheres contained in the reconstructions presented in Aste et al. [12] might therefore have led to errors in the determination of quantities such as density or average number of contact neighbors.

\section{Conclusions}

A high-precision three-dimensional image of a $0.5 \mathrm{~L}$ sample containing about $2 \times 10^{4}$ spheres was reconstructed in less than $1 \mathrm{~h}$ on a standard desktop computer from an MRI tomography acquired in a time shorter than $6 \mathrm{~h}$. A direct measurement of the precision of the technique showed that the errors in the reconstructed data, both in terms of repeatability and accuracy, are comparable to the physical diameter dispersion of the spheres composing the sample, suggesting that better precision could be obtained by reducing the dispersion in sphere diameter. The flawlessness and the high accuracy granted by this method have already proven to be crucial for the discovery of novel properties of highly mono-dispersed sphere packings, allowing accurate determination of the radial two-body correlation function and the observation of important geometrical properties [16].

Acknowledgments R.B. and A.L.S. thank A. Magill for technical help. R.B. thanks S. Franchini and V. Paladino for useful discussions. 
Conflict of interest The authors declare no competing financial interests. This work was supported by the Swiss National Science Foundation (Grants no. PPOOP2-133562 and 200020-126534), the Centre d'Imagerie BioMédicale (CIBM) of the UNIL, UNIGE, HUG, CHUV, EPFL, the Leenards and Jeantet Foundations.

\section{References}

1. L.P. Kadanoff, Rev. Mod. Phys. 71, 435-444 (1999)

2. R.L. Brown, J.C. Richards, P.V. Danckwerts, Principles of Powder Mechanics, 1st edn. (Pergamon Press, Oxford, 1970)

3. R.M. Nedderman, J. Fluid Mech. 286, 405-445 (1995)

4. O. Dauchot, in Ageing and the Glass Transition, ed. by M. Henkel, M. Pleimling, R. Sanctuary (Springer, Berlin, Heidelberg, 2007), pp. 161-206. doi:10.1007/3-540-69684-9

5. H.M. Jaeger, S.R. Nagel, R.P. Behringer, Rev. Mod. Phys. 68, 1259-1273 (1996)

6. T.C. Hales, Disc. Comp. Geo. 36, 5-20 (2011)

7. G.D. Scott, Nature 194, 956-957 (1962)

8. J.D. Bernal, J. Mason, Nature 188, 910-911 (1960)

9. G. Mason, Nature 217, 733-735 (1968)

10. G.D. Scott, D.M. Kilgour, J. Phys. Appl. Phys. 2, 863 (1969)

11. J. Duran, Sands, Powders, and Grains, 1st edn. (Springer, New York, 2000). doi:10.1007/978-14612-0499-2

12. T. Aste, M. Saadatfar, T.J. Senden, Phys. Rev. E 71, 061302 (2005)

13. J.G. Berryman, Phys. Rev. A 27, 1053-1061 (1983)

14. G.Y. Onoda, E.G. Liniger, Phys. Rev. Lett. 64, 2727-2730 (1990)

15. F.M. Schaller, M. Neudecker, M. Saadatfar, G. Delaney, K. Mecke, G.E. Schröder-Turk, M. Schröter, AIP Conf. Proc. 1542, 377-380 (2013). doi:10.1063/1.4811946

16. R. Balzan, A.L. Sellerio, D. Mari, A. Comment, G. Gremaud, Granul. Matter 15, 873-879 (2013)

17. Z.-P. Liang, P.C. Lauterbur, Principles of Magnetic Resonance Imaging: A Signal Processing Perspective, 1st edn. (Wiley-IEEE Press, Oxford, 1999)

18. N. Otsu, IEEE Trans. Syst. Man Cyber. SMC 9, 62-66 (1979)

19. B. Chanda, D.D. Majumder, Digital Image Processing and Analysis (PHI Learning Pvt. Ltd., Delhi, 2004)

20. A. Rosenfeld, ACM Comput. Surv. 1, 147-176 (1969)

21. F. Aurenhammer, ACM Comput. Surv. 23, 345-405 (1991) 